\title{
An Update on the Novel Coronavirus SARS-CoV-2 Pandemic
}

\author{
Subarna Chakravorty MBBS PhD MRCPCH FRCPath \\ Consultant paediatric Haematologist, Kings College Hospital, London, UK
}

\section{Subarna.chakravorty@nhs.net}

Key words

Pandemic, Coronavirus, SARS-CoV-2,

Cite as: Chakravorty S. An Update of the Novel Coronavirus SARS-Cov-2 Pandemic. Sushruta 2020 (March) vol 13; issue 1: ePub 22.03.2020

Full Text

In an unprecedented show of scientific solidarity and collaboration across the globe, research on the novel SARS-CoV-2 (Covid-19) virus has progressed at a pace that could almost match the virus's relentless movement through communities. This has been a pandemic like no other. Whilst it is hard to speculate how the world will emerge at the end of this, there is little doubt that scientists are trying to rise up to its challenge in a way that has never been seen before. As of the $21^{\text {st }}$ of March 2020, the National Library of Medicine research repository has a collection of nearly 1000 articles on Covid-19, a novel virus whose genome was sequenced less than 12 weeks ago ${ }^{12}$. Almost all these articles were published in February and March 2020, reflecting the explosion of global research activity on this virus.

Scientists, physicians, epidemiologists and countless other professional groups are currently engaged in active Covid-19 research. Output of such research is being editorially fast-tracked to ensure maximum dissemination and impact ${ }^{3}$. At the time of this article going to press, the clinicaltrials.gov website has 125 registered clinical trials on COVID-19 treatment, on human subjects, of which 57 trials are currently recruiting ${ }^{4}$.

The internet and social media have facilitated real-time updates on the identification, treatment and outcome of infection in countries all over the world. Political leaders and policy makers are relying on such information to influence human behaviour and contain the infection within populations. In the midst of all this, there is an equally robust and potentially highly dangerous epidemic of high-profile misinformation, outright lies, speculation, conspiracy theories and pseudo-science attempting to derail important scientific advances for political or financial gain.

It is not surprising that the average reader of medical literature might find it confusing and overwhelming to keep track of credible Covid-19 research. Many science writers and science publishing houses have identified this gap and are attempting to keep the public informed of research from established research groups. This article attempts to give the reader an overview of Covid-19 research and signpost them to credible repositories of rigorously curated information of this pandemic. 


\section{Epidemiology:}

Daily updates on new cases and deaths are available from the websites of the Centers for Disease Control and Prevention, (https://www.cdc.gov/), the European Centre for Disease Prevention and Control (https://www.ecdc.europa.eu/en) and the World Health Organisation (WHO) (https://www.who.int/). A carefully curated repository of data derived from the European CDC and other resources are available at https://ourworldindata.org/. The National Health Service in the UK have several useful and well researched information sources. A dashboard of daily cases in the 4 UK nations, including the number of patients who are recovered, is updated by Public Health England (PHE) and can be accessed here. PHE is also providing guidance to the public on self-isolation, information for businesses, employment and financial support, and information public goods and services (https://www.gov.uk/coronavirus).

\section{Pandemic modelling and social policy:}

This has been a controversial issue where scientific evidence and political interest comes into conflict. On the $30^{\text {th }}$ of January 2020, the Governor General of the WHO, Dr Thedros Ghebreyesus declared the Covid-19 disease outbreak a 'public health emergency of international concern' and implored all nation states to consolidate crisis management teams and cooperate with the United Nations' international systems-wide management programme to prepare and respond to the pandemic ${ }^{5}$. Subsequently, the UK has been criticised for its public policy to manage the pandemic by emphasising on contact tracing and containment, defying WHO guidance by delaying social distancing measures and limiting the availability of testing. The UK government has claimed that its social policy is informed by pandemic modelling by a group of experts from Imperial College London. The Imperial paper can be found here- it predicts social isolation models and virus spread trajectories based on the group's previous experience in epidemic modelling and data from from the Wuhan Province in China and North Italy. Unfortunately, the social isolation policy stated in the paper was not implemented for at least 2 crucial weeks in March 2020, which would certainly have contributed to the rising case numbers ${ }^{6}$. Countries of interest to the reader might include China $^{7}$, South Korea ${ }^{8}$, USA (cdc.gov) and India (https://www.mohfw.gov.in/) and the latest WHO reports $(23 / 3 / 20)$ indicates that the virus has now spread to 185 countries, with Southern Europe being its current epicentre.

Despite WHO guidance, public policy appears to vary widely between countries, for example South Korea has invested in widespread population screening and case isolation, and this has paid dividends in curbing the rate at which new cases were being reported earlier in the month. The policy of 'find, isolate, test, treat' proposed by Dr Thedros Ghebreyesus is being adapted by countries in varying degrees of rigour. Initial data appears to support that all four measures are needed for curbing the exponential rise in cases, as demonstrated by the South Korean programme of Covid-19 control ${ }^{9}$. The importance of social distancing is further supported by modelling that has demonstrated that prior to the lockdown imposed in Wuhan Province on $23 / 1 / 20,55 \%$ of people who were being infected by each person remained undetected, and due to their greater numbers and widespread mobility, undocumented/undetected infections were the infection source for nearly $80 \%$ of infected cases ${ }^{10}$. Thus, the policy of only testing people with history of travel to Europe or China, or 
those with symptoms only may not be sufficient in halting its spread. Social distancing and widespread testing are crucial steps in limiting its exponential spread.

\section{Clinical data:}

Initial case reports from China and subsequently from Italy has informed the rest of the world on how to prepare for the pandemic. Data indicate that human to human transmission ${ }^{11}$ occur via aerosol and fomites ${ }^{12}$ and that age (median age 45-60 years years), gender (male > female) and pre-existing conditions (hypertension and diabetes) were important risk factors 13. Cough and fever were common symptoms, computerised tomograph of chest showed typical pneumonic features, and lymphopaenia was near universal.

Mortality estimates vary from 1.5 to $3.6 \%$ when based on the number of deaths relative to the number of confirmed cases of infection ${ }^{13}$. However, some experts have argued that this figure is based on the number of deaths relative to the number of confirmed cases of infection on the day of the person's death. They have argued that this was not representative of the actual death rate, as many more people are likely to have been infected at the time a person dies of Covid-19 compared to the day when the person was infected. Epidemiological data suggest that the maximum incubation period is 14 days, hence the denominator to calculate death rates should be the number of infected individuals 14 days prior to the day of death. As of $1 / 3 / 2020$, recalculating death rates using this method resulted in significantly higher values $5.6 \%$ (95\% confidence interval 5.4-5.8) for China and $15 \cdot 2 \%(12 \cdot 5-17 \cdot 9)$ outside of China ${ }^{14}$. Additionally, mortality was very high among the minority of patients who had severe disease, and early reports from China indicate $62 \%$ critically ill patients and $82 \%$ needing mechanical ventilation died ${ }^{15}$. In contrast, the disease course in children was significantly mild, and in one case series from Wuhan, only those with underlying conditions ( 3 out of 172, $1.8 \%)$ needed mechanical ventilation, one of whom died. Importantly, majority of children (approximately $60 \%$ ) had no fever at presentation, and $15 \%$ of those who tested positive were asymptomatic ${ }^{16}$.

\section{Treatment:}

A huge amount of data has been systematically collected and shared by Chinese clinicians to allow planning of effective interventions. Information on human to human transmission, high rates of intra-familial spread, evidence of faecal oral transmission, relatively mild clinical course in children, have all arisen from painstakingly collected clinical data ${ }^{11}$ amidst huge operational challenges of managing an epidemic. These have informed policy making internationally. Additionally, host susceptibility data have allowed better resource planning in epidemic preparedness ${ }^{17}$. In order to provide targeted treatment to the most at risk of developing severe disease, critical care clinicians have developed clinical risk scoring based on several parameters including age, attendant co-morbidities, lymphocyte count at presentation, respiratory rate among others. This has enabled early recognition and appropriate triage of patients toward continuous or intermittent monitoring, resulting in judicious use of resources and have resulted in improved outcome ${ }^{18}$.

A vast number of existing pharmaceutical products are being used and tested in Covid-19. A large number of antiviral agents including ribavirin, oseltamivir and others were empirically 
given to patients with pneumonia in most centres. A large, randomised controlled trial of the known HIV combination drug lopinavir/ritonavir in 199 hypoxic patients with laboratoryconfirmed Covid-19 demonstrated no additional benefit compared to standard supportive care ${ }^{19}$. Remdesivir, broad -spectrum antiviral drug with efficacy against Ebola virus has also shown some promise ${ }^{20}$ and is currently being trialled in human subjects ${ }^{4}$. Chloroquine has gained widespread recognition as a potential therapeutic option. Due to its generic production status, low cost and oral availability, it is of significant interest among clinicians and the general public. Both remdesivir and chloroquine have demonstrated in vitro inhibition of SARS-CoV2 ${ }^{21}$. An open label, non-random clinical study using hydroxychloroquine and azithromycin has shown some feasibility of its use in Covid-19 ${ }^{22}$, but well-designed randomised controlled trials are needed before drugs with substantial adverse effects are released for indiscriminate use ${ }^{23}$.

\section{Prevention:}

A vaccine against the SARS-CoV2 virus is urgently needed to stop its spread in the community and a large number of laboratories around the globe are attempting to produce a vaccine which is both safe and efficacious and several human trials have already started. Whilst a vaccine development is likely to be the only way the epidemic will be finally controlled, it is important to ensure adequate safety checks are in place and sufficient pre-clinical animal testing is undertaken to carefully study host response and adverse effects of these vaccines 24 .

\section{Important research questions:}

- Does the virus result in immune memory sufficient to provide long term protection from reinfection?

- How does virulence change following several passages of human to human transmission?

- How quickly can a reliable serological test be made available to study the true infection rates in affected communities?

- What drugs and vaccines will show promise?

- What new molecules can combat severe host immune response to Covid-19?

- Are there genetically determined modifiers of human susceptibility to virus, host response and response to therapy?

- What are the best strategies to protect healthcare workers caring for Covid -19 patients who have to undertake aerosol -generating procedures?

\section{Covid-19 preparedness in the UK}

The infection and death rate reports from Italy have served as a sombre reminder to doctors and policy makers in the UK of the seriousness of this pandemic and have helped in the preparedness of institutions, businesses and healthcare providers, occasionally in advance of government decrees ${ }^{6}$. It is likely that the UK health system will be severely stretched with rising number of severely affected adults needing critical care. Severe scarcity of resources has forced Italian doctors to deploy rationing methods which may pose significant ethical challenges. It may be necessary to remove such difficult triage decisions away from treating physicians and into the hands to hospital managers with defined criteria for treatment escalation ${ }^{25}$. 


\section{Who to follow on Social Media?}

Misinformation and speculation is rife in the current pandemic. Important scientific and policy-related information can be found in social media, and Twitter is an invaluable source for information. The WHO (@WHO),CDC (@CDCgov), Public Health England (@PHE_UK) have twitter handles that are worth following for up to date information on policy and data. Several thought leaders have emerged in this pandemic who have worked tirelessly to debunk myths, misinformation and propaganda- follow people like @jpogue1, @devisridhar, @edyong209, @imartincorena, @richardhorton1, @maxcroser, @bhalomanush for insightful analyses.

\section{Conclusion:}

It is likely that the Covid-19 pandemic will change the world forever- in ways we work and support each other, the way nation states define its health policies, how out NHS responds to the severe constraints in resources- the chinks in the NHS armour are already showing. However, even in these bleak dystopian days, we hear of stories of enormous courage and fortitude, stories which will inspire generations to come and help us rebuild our lives when all this blows over. 


\section{References:}

1. Wu F, Zhao S, Yu B, et al. A new coronavirus associated with human respiratory disease in China. Nature 2020;579:265-9.

2. Coronavirus Covid-19 articles. 2020. (Accessed 21/3/2020, 2020, at https://www.ncbi.nlm.nih.gov/pubmed/?term=coronavirus+covid-19.)

3. Rubin EJ, Baden LR, Morrissey S, Campion EW. Medical Journals and the 2019-nCoV Outbreak. N Engl J Med 2020;382:866.

4. Covid-19 trials registered on clinicaltrials.gov. 2020. (Accessed 21/03/2020, 2020, at https://clinicaltrials.gov/ct2/results?cond=covid-19\&term=\&cntry=\&state=\&city=\&dist=.)

5. World Health Organisation Covid-19 Response. 2020. (Accessed 22/3/2020, 2020, at https://covid-19-response.org/.)

6. Hunter DJ. Covid-19 and the Stiff Upper Lip - The Pandemic Response in the United Kingdom. N Engl J Med 2020.

7. Zhou $P$, Yang $X L$, Wang $X G$, et al. A pneumonia outbreak associated with a new coronavirus of probable bat origin. Nature 2020;579:270-3.

8. Choi SC, Ki M. Estimating the reproductive number and the outbreak size of Novel Coronavirus disease (COVID-19) using mathematical model in Republic of Korea. Epidemiol Health 2020:e2020011.

9. Normile D. Coronavirus cases have dropped sharply in South Korea. What's the secret to its success? Science2020.

10. Li R, Pei S, Chen B, et al. Substantial undocumented infection facilitates the rapid dissemination of novel coronavirus (SARS-CoV2). Science 2020.

11. Chan JF, Yuan S, Kok KH, et al. A familial cluster of pneumonia associated with the 2019 novel coronavirus indicating person-to-person transmission: a study of a family cluster. Lancet 2020;395:514-23.

12. van Doremalen N, Bushmaker $\mathrm{T}$, Morris $\mathrm{DH}$, et al. Aerosol and Surface Stability of SARS-CoV-2 as Compared with SARS-CoV-1. N Engl J Med 2020.

13. Guan WJ, Ni ZY, Hu Y, et al. Clinical Characteristics of Coronavirus Disease 2019 in China. N Engl J Med 2020.

14. Baud D, Qi X, Nielsen-Saines K, Musso D, Pomar L, Favre G. Real estimates of mortality following COVID-19 infection. Lancet Infect Dis 2020.

15. Yang $X, Y u Y, X u$ J, et al. Clinical course and outcomes of critically ill patients with SARSCoV-2 pneumonia in Wuhan, China: a single-centered, retrospective, observational study. Lancet Respir Med 2020.

16. Lu X, Zhang L, Du H, et al. SARS-CoV-2 Infection in Children. N Engl J Med 2020.

17. Shi Y, Yu X, Zhao H, Wang H, Zhao R, Sheng J. Host susceptibility to severe COVID-19 and establishment of a host risk score: findings of 487 cases outside Wuhan. Crit Care 2020;24:108.

18. Sun Q, Qiu H, Huang M, Yang Y. Lower mortality of COVID-19 by early recognition and intervention: experience from Jiangsu Province. Ann Intensive Care 2020;10:33.

19. Cao B, Wang Y, Wen D, et al. A Trial of Lopinavir-Ritonavir in Adults Hospitalized with Severe Covid-19. N Engl J Med 2020.

20. Ko WC, Rolain JM, Lee NY, et al. Arguments in favour of remdesivir for treating SARSCoV-2 infections. Int J Antimicrob Agents 2020:105933.

21. Wang $M$, Cao $R$, Zhang $L$, et al. Remdesivir and chloroquine effectively inhibit the recently emerged novel coronavirus (2019-nCoV) in vitro. Cell Res 2020;30:269-71. 
22. Gautret $\mathrm{P}$, Lagier, JC., Parola, P, et al.,. Hydroxychloroquine and azithromycin as a treatment of

COVID-19: results of an open-label non-randomized clinical trial. International Journal of Antimicrobial Agents 2020.

23. Cortegiani A, Ingoglia G, Ippolito M, Giarratano A, Einav S. A systematic review on the efficacy and safety of chloroquine for the treatment of COVID-19. J Crit Care 2020.

24. Jiang S. Don't rush to deploy COVID-19 vaccines and drugs without sufficient safety guarantees. Nature 2020;579:321.

25. Rosenbaum L. Facing Covid-19 in Italy - Ethics, Logistics, and Therapeutics on the Epidemic's Front Line. N Engl J Med 2020. 